\title{
OMAE 2011-49117
}

\section{MODELLING OF THE IPS BUOY WAVE ENERGY CONVERTER INCLUDING THE EFFECT OF NON-UNIFORM TUBE CROSS-SECTION}

\author{
António F. O. Falcão ${ }^{1}$, José J. M. B. Cândido ${ }^{1}$, Paulo A. P. Justino ${ }^{2}$, João C. C. Henriques ${ }^{1}$ \\ ${ }^{1}$ IDMEC, Instituto Superior Técnico, Technical University of Lisbon, 1049-001 Lisbon, Portugal \\ ${ }^{2}$ Laboratório Nacional de Energia e Geologia, Estrada Paço do Lumiar, 1649-038 Lisbon, Portugal \\ E-mails: antonio.falcao@ist.utl.pt; josé.candido@ist.utl.pt; paulo.justino@ineti.pt; joaochenriques@ist.utl.pt
}

\begin{abstract}
An important class of floating wave energy converters (that includes the IPS buoy, the Wavebob and the PowerBuoy) comprehends devices in which the energy is converted from the relative (essentially heaving) motion between two bodies oscillating differently. The paper considers the case of the IPS buoy, consisting of a floater rigidly connected to a fully submerged vertical (acceleration) tube open at both ends. The tube contains a piston whose motion relative to the floater-tube system (motion originated by wave action on the floater and by the inertia of the water enclosed in the tube) drives a power take-off mechanism (PTO) (assumed to be a linear damper). To solve the problem of the end-stops, the central part of the tube, along which the piston slides, bells out at either end to limit the stroke of the piston. The use of a hydraulic turbine inside the tube is examined as an alternative to the piston. A frequency domain analysis of the device in regular waves is developed, combined with a one-dimensional unsteady flow model inside the tube (whose cross-section is in general nonuniform). Numerical results are presented for a cylindrical buoy in regular waves, including the optimization of the acceleration tube geometry and PTO damping coefficient for several wave periods.
\end{abstract}

\section{Introduction}

The concept of the point absorber for wave energy utilization was developed in the late 1970s and early 1980s, mostly in Scandinavia [1]. This is in general a wave energy converter of oscillating body type whose horizontal dimensions are small compared to the representative wave length. In its simplest version, the body reacts against the bottom. In deep water (say $50 \mathrm{~m}$ or more), this may raise difficulties due to the distance between the floating body and the sea bottom. Multibody systems may then be used instead, in which the energy is converted from the relative motion between two bodies oscillating differently. This is the case of several devices presently under development, like the Pelamis, the Wavebob and the PowerBuoy.

Sometimes the relevant relative motion results from heaving oscillations. This paper considers the special situation when a floater reacts against the inertia of the water contained in a long vertical tube open at both ends, located underneath. This is the case of the spar-buoy OWC, possibly the simplest concept for a floating oscillating water column (OWC) device equipped with an air turbine, in which the upper end of the tube extends through the buoy above the sea water level. Yoshio Masuda, in Japan developed a navigation buoy based on the OWC spar buoy concept $[2,3]$. The spar-buoy OWC will not be analysed in this paper.

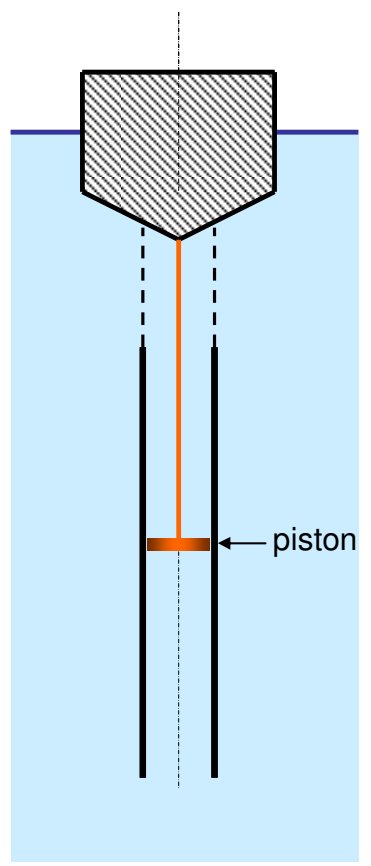

Fig. 1. Schematic representation of the IPS buoy. 
The IPS buoy is another type of spar-buoy and will be analysed here in detail. It was invented by Sven A. Noren [4] and initially developed in Sweden by the company Interproject Service (IPS). The device consists of a buoy rigidly connected to a fully submerged vertical tube (the so-called acceleration tube) open at both ends (Fig. 1). The tube contains a piston whose motion relative to the floater-tube system (motion originated by wave action on the floater and by the inertia of the water enclosed in the tube) drives a power take-off (PTO) mechanism. The same inventor later introduced an improvement that significantly contributes to solve the problem of the end-stops: the central part of the tube, along which the piston slides, bells out at either end to limit the stroke of the piston [5].

A half-scale prototype of the IPS buoy was tested in sea trials in Sweden, in the early 1980s [6]. The AquaBuOY is a wave energy converter, developed in the 2000s, that combines the IPS buoy concept with a pair of hose pumps to produce a flow of water at high pressure that drives a Pelton turbine $[7,8]$. A prototype of the AquaBuOY was deployed and tested in 2007 in the Pacific Ocean off the coast of Oregon.

A variant of the initial IPS buoy concept, due to Stephen Salter, is the sloped IPS buoy: the natural frequency of the converter may be reduced, and in this way the capture width enlarged, if the buoy-tube set is made to oscillate at an angle intermediate between the heave and the surge directions. The sloped IPS buoy has been studied since the mid-1990s at the University of Edinburgh, by model testing and numerical modelling [9-11].

\section{Theoretical modelling}

The IPS buoy consists basically of a buoy rigidly connected to a fully submerged tube (the acceleration tube), oscillating in heave, by the action of the waves, with respect to a piston that can slide along the tube. The wave energy is absorbed by means of the relative motion between the piston and the buoytube set. The concept is represented in Fig. 1.

We note that most of the inertia against which the buoytube set moves is that of the water contained inside the acceleration tube (obviously in addition to the mass of the piston itself). In the simplified mathematical modelling adopted in this paper, we assume that the buoy-tube set is constrained to oscillate in heave, an assumption that seems reasonable taking into account the axial extent of the device.

We introduce the following assumptions. (i) The tube is sufficiently far away underneath the buoy for the hydrodynamic interaction between both to be negligible. (ii) The interaction between the wave fields induced by the two ends of the tube may be neglected. (iii) The distance from the free surface to the tube upper end is large enough for the excitation and radiation forces on the flow about the two tube ends to be neglected. (We note however that the added mass at the two ends of the tube will be accounted for.) (iv) Finally, the flow inside the tube is modelled as one-dimensional. Admittedly, some of these simplifications may be rather drastic. This is specially the case of assumptions (i) and (iii) if the distances from the acceleration tube to the buoy and to the free surface are not large enough. In spite of this, the present paper is expected to provide useful insights into the relationships between device geometry, PTO parameters and wave energy converter performance. Naturally, in cases of special practical interest, this simplified approach should be complemented by a more rigorous analysis using tools like the commercially available codes (e.g. WAMIT, AQUADYN) based on the boundary element method for the computation of the hydrodynamic coefficients, including the interference between the buoy and the acceleration tube, as done in [12].

2.1 One-dimensional flow inside the tube. We consider now the flow inside the acceleration tube, whose total length is $L$ (Fig. 2). The position of the tube

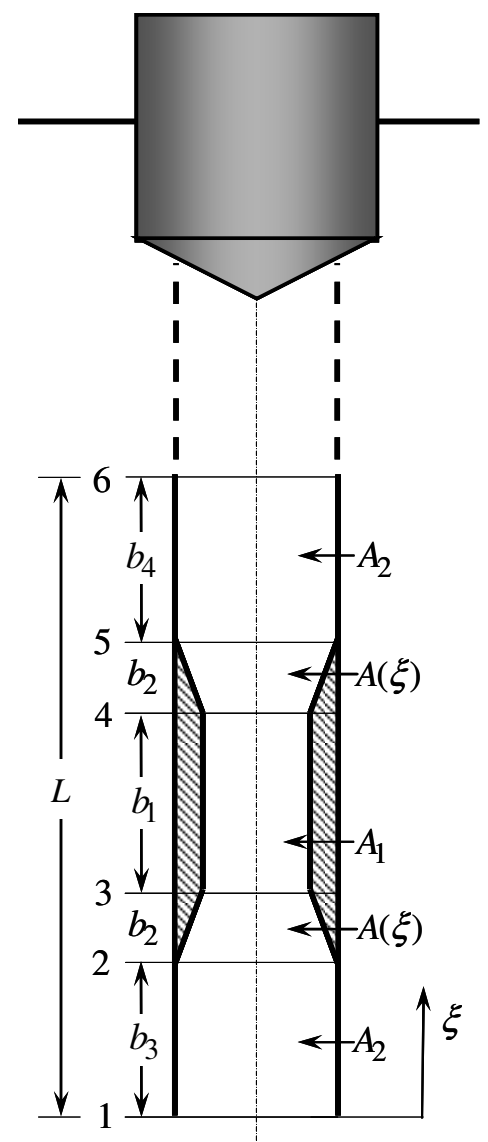

Fig. 2. IPS buoy with acceleration tube.

sections are defined by a longitudinal coordinate $\xi$ (with $\xi=0$ at the lower end of the tube). The piston is allowed to move, relative to the tube, inside a central part 3-4 (working part), $\quad b_{2}+b_{3} \leq \xi \leq b_{1}+b_{2}+b_{3}$, of length $b_{1}$ and cross sectional area $A_{1}$, as shown in Fig. 2. The working part is continued downwards and upwards by tube parts $1-2$ and 5-6, 
of lengths $b_{3}$ and $b_{4}$, respectively, both of cross sectional area $A_{2}=\alpha^{2} A_{1} \quad(\alpha \geq 1)$. The transitions are provided by conical connections 2-3 and 4-5, of cross sectional areas $A(\xi)$. If $\alpha>1$, there may be a significant axial force on the tube resulting from the pressure distribution on the inner conical walls.

The added mass of the oscillating water column contained in a semi-infinite open tube of radius $r$ and of negligible wall thickness in an unbounded perfect fluid is $\rho \pi l r^{2}$, where $l=0.6133 r$ (see $[13,14]$ ). We assume this result to apply to our case (small tube thickness close to the tube ends), with $\pi r^{2}=A_{2}$.

In a fixed frame of reference, the buoy-tube pair moves along its own vertical axis with velocity $W(t)$ (positive for upward motion), where $t$ is time. We note that the water flow inside the tube is unsteady in any referential. In our analysis, we adopt a non-inertial frame of reference fixed to the buoytube pair. In this referential, the piston velocity is $V(t)$ and the one-dimensional flow velocity at a section $\xi$ of area $A(\xi)$ is

$$
v(\xi, t)=\frac{A_{1}}{A(\xi)} V(t) .
$$

For a conical transition, it is

$$
\begin{gathered}
\frac{A(\xi)}{A_{1}}=\left[(\alpha-1) \frac{b_{3}-\xi}{b_{2}}+\alpha\right]^{2} \quad\left(b_{3} \leq \xi \leq b_{2}+b_{3}\right), \\
\frac{A(\xi)}{A_{1}}=\left[(\alpha-1) \frac{\xi-b_{1}-b_{2}-b_{3}}{b_{2}}+1\right]^{2} \\
\left(b_{1}+b_{2}+b_{3} \leq \xi \leq b_{1}+2 b_{2}+b_{3}\right) .
\end{gathered}
$$

In what follows, we assume that the piston is of negligible length and mass (this is equivalent to assuming that its length is non-zero and its mean density is equal to water density). Since the tube is totally submerged, the net force on the piston is not affected by gravity and so we simply ignore the acceleration of gravity for its calculation and denote by $p_{\text {out }}$ the uniform pressure of the supposedly unbounded water far away from the tube ends.

Let $\xi=z$ be the instantaneous position of the piston (assumed of negligible length). Applying Bernoulli's equation for unsteady flow (see e.g. [15]), we find, for the pressure at a section $\xi<z$ below the piston,

$$
\begin{aligned}
p(\xi, t)= & p_{\text {out }}+\rho\left[\alpha^{-2}-\frac{A_{1}^{2}}{A(\xi)^{2}}\right] \frac{V^{2}}{2}-\rho(\xi+l) \frac{d W}{d t} \\
& -\rho \int_{-l}^{\xi} \frac{\partial v(\xi, t)}{\partial t} d \xi
\end{aligned}
$$

The third term on the right-hand-side of Eq. (4) is due to the fictitious body force per unit mass $-d W / d t$ associated with the non-inertial frame of reference. The last term results from the unsteadiness of the velocity $v(\xi, t)$ and may be written as

$$
\begin{gathered}
-\rho \int_{-l}^{\xi} \frac{\partial v(\xi, t)}{\partial t} d \xi=\rho \frac{d V}{d t}\left[\frac{l+b_{3}}{\alpha^{2}}+\frac{b_{2}\left(\xi-b_{3}\right)}{\alpha\left(\alpha b_{2}-b_{3}+\alpha b_{3}+\xi-\alpha \xi\right)}\right] \\
\left(b_{3} \leq \xi \leq b_{2}+b_{3}\right), \\
-\rho \int_{-l}^{\xi} \frac{\partial v(\xi, t)}{\partial t} d \xi=\rho \frac{d V}{d t}\left[\frac{l+b_{3}}{\alpha^{2}}+\frac{b_{2}}{\alpha}+\xi-b_{2}-b_{3}\right] \\
\left(b_{2}+b_{3} \leq \xi \leq z\right) .
\end{gathered}
$$

Above the piston, $\xi>z$, we have

$$
\begin{aligned}
p(\xi, t) & =p_{\text {out }}+\rho\left(\alpha^{-2}-\frac{A_{1}^{2}}{A(\xi)^{2}}\right) \frac{V^{2}}{2} \\
& +\rho(L-\xi+l) \frac{d W}{d t}+\rho \int_{\xi}^{L+l} \frac{\partial v(\xi, t)}{\partial t} d \xi .
\end{aligned}
$$

Expressions similar to $(5,6)$ can be derived for the last term of Eq. (7).

The force on the piston is $f_{p}(t)=A_{1}\left(p\left(z^{-}, t\right)-p\left(z^{+}, t\right)\right)$, where $z^{-}$and $z^{+}$are the coordinates of the lower and upper surfaces of the piston. We find

$$
f_{p}(t)=-M_{W} \frac{d W}{d t}-M_{V} \frac{d V}{d t},
$$

where

$$
\begin{gathered}
M_{W}=\rho A_{1}\left(b_{5}+2 l\right), \\
M_{V}=\rho A_{1}\left(b_{1}+\alpha^{-2}\left(b_{3}+b_{4}+2 l\right)+2 b_{2} \alpha^{-1}\right) .
\end{gathered}
$$

The total axial force $f_{t}(t)$ on the internal surface of the two conical parts of the acceleration tube is

$$
f_{t}(t)=\int_{b_{3}}^{b_{2}+b_{3}} p(\xi, t) \frac{d A(\xi)}{d \xi} d \xi-\int_{L-b_{4}-b_{2}}^{L-b_{4}} p(\xi, t) \frac{d A(\xi)}{d \xi} d \xi .
$$

It may be written as

$$
f_{t}(t)=-m_{W} \frac{d W}{d t}-m_{V} \frac{d V}{d t},
$$

where

$$
\begin{gathered}
m_{W}=\rho A_{1}\left[\left(\alpha^{2}+\alpha-2\right) \frac{2 b_{2}}{3}+\left(\alpha^{2}-1\right)\left(b_{3}+b_{4}+2 l\right)\right], \\
m_{V}=\rho A_{1}\left[2\left(1-\alpha^{-1}\right) b_{2}+\left(1-\alpha^{-2}\right)\left(b_{3}+b_{4}+2 l\right)\right] .
\end{gathered}
$$

The preceding equations show that the forces on the piston, $f_{p}$, and on the tube, $f_{t}$, depend on the sum $b_{3}+b_{4}$, not on the lengths $b_{3}$ or $b_{4}$ separately, a result that is not unexpected. They also show that the expressions of those forces are linear in the accelerations $d W / d t$ and $d V / d t$, with no dependence on velocities. From the viewpoint of the axial force $f_{t}$ on the tube, $m_{W}$ may be regarded as an inertial mass associated with 
the tube (and floater) acceleration $d W / d t$; the same applies to $m_{V}$ in connection with the acceleration $d V / d t$ of the piston in the frame of reference fixed to the buoy-tube pair. If the whole tube is of uniform inner cross section, i.e. if $\alpha=1$, it is simply $M_{V}=M_{W}$ and $f_{t}=m_{V}=m_{W}=0$, a situation that was studied in [16].

2.2 Piston versus hydraulic turbine. The original IPS buoy was conceived with a piston, sliding along the acceleration tube, whose relative motion activates a secondary hydraulic ram (or linear pump) that supplies high pressure liquid (water or oil) to a hydraulic circuit [4]. If energy is to be absorbed from large amplitude waves, the excursion of the primary piston is also relatively large which requires a long rod (possibly longer than $20 \mathrm{~m}$ ), which, when subjected to compression forces, can cause serious buckling problems. An alternative to the piston pump is a pair of hose pumps as in the Aquabuoy [7,8], which avoids compression loads but whose hydraulic circuit working pressure is much lower than what is attainable by piston pumps.

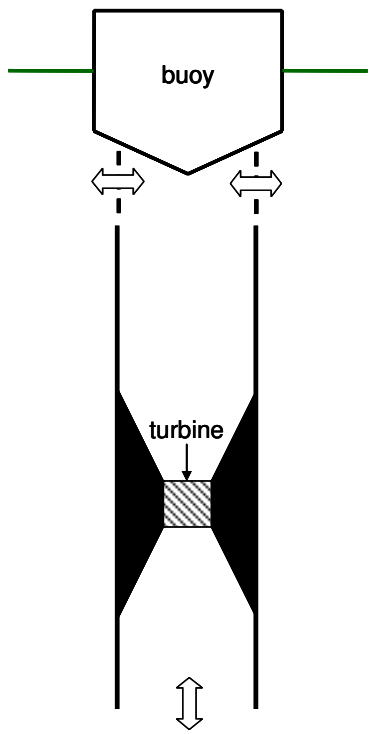

Fig. 3. IPS buoy with the piston replaced by a hydraulic turbine (in the shaded space).

A self-rectifying hydraulic turbine located in the narrower part of the tube may be used instead of a piston, although this seems not to have been proposed before. This avoids the problem of limiting the piston excursion. In order to avoid cavitation, the turbine should be deeply submerged. Naturally, the flow through the turbine is far more complex than the (assumed one-dimensional) flow in the tube. However, for the purpose of accounting for the inertia of the flow through the turbine, we may define an equivalent tube element of length $b_{1}$ and an equivalent cross sectional area $A_{1}$, as shown by the shaded area in Fig. 3. Since the diameter of the turbine is expected to be much smaller than the diameter of the main part of the acceleration tube, the area ratio $\alpha^{2}=A_{2} / A_{1}$ should be much larger than unity. Obviously, the expressions derived above for the piston in tube may be applied to this case, the volume flow rate through the turbine being $Q(t)=A_{1} V(t)$; the turbine pressure head is $\Delta p=f_{p}(t) / A_{1}$, where $f_{p}$ is given by Eq. (8).

2.3 Hydrodynamic analysis in regular waves. We consider now the IPS buoy, and denote by $x(t)$ the coordinate for the heaving motions of the floater-tube set, with $x=0$ in the absence of waves and $x$ increasing upwards. Let $y(t)$ be the oscillations in piston position relative to the buoy-tube set. We note that it is $\dot{x}=W$ and $\dot{y}=V$, where $W$ is the tube velocity and $V$ is the relative velocity of the piston as defined in subsection 2.1. We consider a linear PTO such that a relationship

$$
f_{p}=K y+C \dot{y}
$$

holds between the force on the piston $f_{p}$ and the relative displacement $y$ and velocity $\dot{y}$ of the piston. The constant $C$ is the PTO damping coefficient and the constant $K$ may be regarded as a spring stiffness. The instantaneous power absorbed by the PTO is $P=f_{p} \dot{y}$.

The following hydrodynamic analysis is based on linear water wave theory, which, as is well known, requires the wave amplitude and the amplitude of body oscillations to be small (compared with wavelength). The equation of motion can be found in [17]. We consider the body to consist of a floater (subscript $1 a$ ) and a tube (subscript $1 b$ ), and denote by $m_{1 a}$, $m_{1 b}$ and $\mu_{1 a}, \mu_{1 b}$ the corresponding masses and added masses. The added mass $\mu_{1 b}$ of the tube is supposed to be independent from the wave frequency (as in an unbounded medium).

Provided that the PTO is linear (as assumed above) and after the transients related to the initial conditions have died out, we may write, for the motion of the body in the presence of incoming sinusoidal waves of frequency $\omega$,

$$
\left(M_{1 a}+M_{1 b}\right) \ddot{x}+B \dot{x}+\rho g S x=f_{e}(t)+f_{t}(t)+f_{p}(t) .
$$

Here $M_{1 a}=m_{1 a}+\mu_{1 a}$ and $M_{1 b}=m_{1 b}+\mu_{1 b}$ are the mass plus added mass of bodies $1 a$ and $1 b$, respectively, $\rho$ is water density, $g$ is acceleration of gravity, $B(\omega)$ is radiation damping coefficient of the buoy (body $1 a$ ), and $S$ is the cross sectional area of the floater defined by the undisturbed free-surface. We note that $\mu_{1 a}$ is a function of $\omega$ and recall that it is $\dot{x}=W$, where $W$ is the tube velocity defined in subsection 2.1 . On the right-hand-side of Eq. (16), $f_{e}$ is the hydrodynamic excitation force on the floater due to the incoming waves, $f_{t}$ is the force on the inner surface of the tube given by Eq. (12) and $f_{p}$ is the vertical force on the piston given by Eq. (8). We recall that 
negligible hydrodynamic interference is assumed. We note also that $\mu_{1 b}$ is supposed not to be a function of frequency $\omega$ (as a consequence from the assumption of deep submergence of body $1 b)$.

Since we have a linear system acted upon by a simpletime-harmonic excitation force of frequency $\omega$, we may write, after the transients related to the initial conditions have died out,

$$
\left\{x, y, V, f_{e}\right\}=\left\{X, Y, V_{0}, F_{e}\right\} e^{i \omega t} .
$$

Here $X, Y, V_{0}=i \omega Y$ and $F_{e}$ are complex amplitudes. We may write $F_{e}=A_{w} \Gamma(\omega)$, where $A_{w}$ is the incident wave (real) amplitude, and $\Gamma$ is the (in general complex) excitation force coefficient. The absolute value of $\Gamma(\omega)$ may be related to $B(\omega)$ by the Haskind relation (valid for an axisymmetric body oscillating in heave in deep water, see [17])

$$
|\Gamma(\omega)|=\left[\frac{2 g^{3} \rho B(\omega)}{\omega^{3}}\right]^{1 / 2} .
$$

By using the complex amplitude representation, we easily obtain, from Eqs (8), (12) (16) and (17),

$$
\begin{gathered}
-\omega^{2}\left(M_{1 a}+M_{1 b}+m_{W}+M_{W}\right) X+i \omega B X+\rho g S X \\
-\omega^{2}\left(m_{V}+M_{V}\right) Y=F_{e}, \\
\omega^{2} M_{W} X+\omega^{2} M_{V} Y=(K+i \omega C) Y .
\end{gathered}
$$

For given wave frequency $\omega$ and excitation force amplitude $F_{e}$, the pair of linear algebraic equations (19) and (20) yield the complex amplitudes $X$ and $Y$. The time-averaged value of the power absorbed by the PTO (piston or turbine) is $\bar{P}=\omega^{2} C|Y|^{2} / 2$.

If the whole tube is of uniform inner cross section, i.e. if $\alpha=1, \quad$ it is simply $A_{1}=A_{2}, \quad f_{t}=m_{V}=m_{W}=0 \quad$ and $M_{V}=M_{W}=M_{2}$ (say), where $M_{2}$ is the mass plus added mass of the water contained in the tube. In this case, Eqs (19, 20) reduce to

$$
\begin{array}{r}
-\omega^{2}\left(M_{1 a}+M_{1 b}+M_{2}\right) X+i \omega B X \\
+\rho g S X-\omega^{2} M_{2} Y=F_{e}, \\
\omega^{2} M_{2} X+\omega^{2} M_{2} Y=(K+i \omega C) Y .
\end{array}
$$

Since we only consider heave oscillations, the equations of motion are not affected by how the mass $m_{1}=m_{1 a}+m_{1 b}$ is distributed between bodies $1 a$ and $1 b$. For convenience of presentation of numerical results, we assume that $m_{1 a}$ is the mass of water of volume equal to the submerged part of the buoy in the absence of waves.

\section{Numerical results}

Numerical results were obtained for a cylindrical buoy of radius $a$, with a conical bottom (semi-angle of the cone equal to $\pi / 3)$. In calm water, the cylindrical part of the buoy is submerged to a depth equal to the radius $a$. The added mass $\mu_{1 a}$ and the radiation damping coefficient $B$ were computed with the software WAMIT for a set of values of the frequency $\omega$ and deep water. A dimensionless plot of $\mu_{1 a}^{*}=\mu_{1 a} /\left(\rho \pi a^{3}\right)$ and $\quad B^{*}=B /\left(\rho \pi a^{3} \omega\right) \quad$ versus $\quad T^{*}=T(g / a)^{1 / 2} \quad(T=$ wave period) is shown in Fig. 4 . We define the dimensionless value of the mass plus added mass of body $1 b$ as $M_{1 b}^{*}=M_{1 b} / m_{1 a}$, where, for the geometry considered here, it is $m_{1 a}=3.746 \rho a^{3}$ In the special case when $\alpha=1$, i.e. an acceleration tube of uniform inner cross section $\left(A_{1}=A_{2}\right)$, we also define the dimensionless mass plus added mass of body 2 (water contained in the tube) as $M_{2}^{*}=M_{2} / m_{1 a}$, where $M_{2}=\rho A_{1}(L+2 l)$.

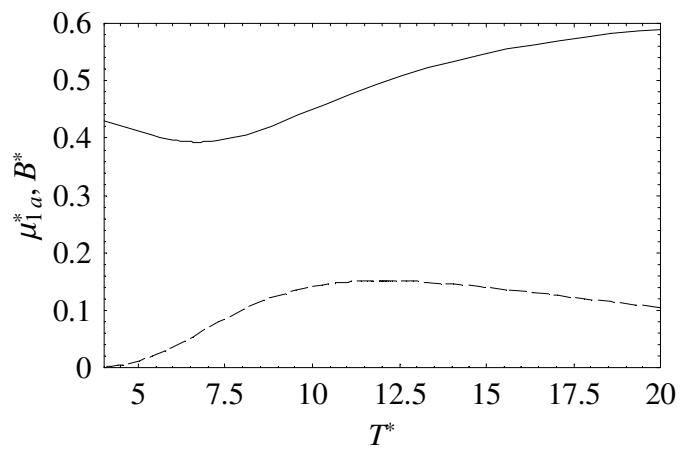

Fig. 4. Dimensionless plot of the added mass $\mu_{1 a}^{*}$ (solid line) and radiation damping coefficient $B^{*}$ (dotted line) versus wave period $T^{*}$, for the buoy (body $1 a$ ) in deep water.

Here, we consider regular waves of frequency $\omega$ and assume that the PTO consists solely of a linear damper (no spring, i.e. $K=0)$. We define a dimensionless damping coefficient $C^{*}(\omega)=C / B(\omega)$, where $B(\omega)$ is the radiation damping coefficient of body $1 a$. We also define dimensionless values $X_{0}^{*}=|X| / A_{w} \quad\left(A_{w}=\right.$ incident wave amplitude $)$ for the motion amplitude of body 1 (buoy-tube set) and $Y^{*}=|Y / X|$ for the amplitude of the piston motion relative to the buoy-tube pair. Note that $Y^{*}=0$ means that the piston is rigidly connected to the buoy. If the piston does not move (possibly because the inertia of the water inside the tube is infinite) it is $\dot{x}=-\dot{y}$ and $Y^{*}=1$.

The theoretical maximum limit for the time-averaged wavepower that can be absorbed from regular waves in deep water by a heaving wave energy converter with a vertical axis of symmetry is well known to be (see [17])

$$
\bar{P}_{\max }=\frac{g^{3} \rho A_{w}^{2}}{4 \omega^{3}} .
$$


Accordingly we define the dimensionless power $\vec{P}^{*}=\bar{P} / \bar{P}_{\max } \leq 1$, where $\bar{P}=\omega^{2} C|Y|^{2} / 2$ is the time-averaged power absorbed from the waves.

3.1 Tube of uniform cross section $\alpha=1$. We consider first the case when $\alpha=1$ (and $f_{t}=0$ ). Since the inner cross section of the tube is uniform, the flow of water (assumed onedimensional) inside the acceleration tube is also uniform (the water moves as a solid body) and the system is equivalent to a two-body heaving wave energy converter in which the mass plus added mass of bodies 1 and 2 are respectively $M_{1 a}+M_{1 b}$ and $M_{2}$, and the PTO is activated by the relative motion between the bodies. This two-body case was theoretically analysed in detail by Falnes [18].

An optimization was performed that consisted in finding the pair of dimensionless values $C^{*}$ and $M_{2}^{*}$ that maximizes $\bar{P}^{*}$, for given values of the dimensionless wave period $T *$ and of $M_{1 b}^{*}$. This two-dimensional optimization was performed with the aid of the FindMaximum subroutine of Mathematica. Results are shown in Figs 5-7 for $T^{*}=10,12$ and 14. The following curves (dimensionless values) are plotted (versus $M_{1 b}^{*}$ ): (i) amplitude $Y^{*}=|Y / X|$ of the relative
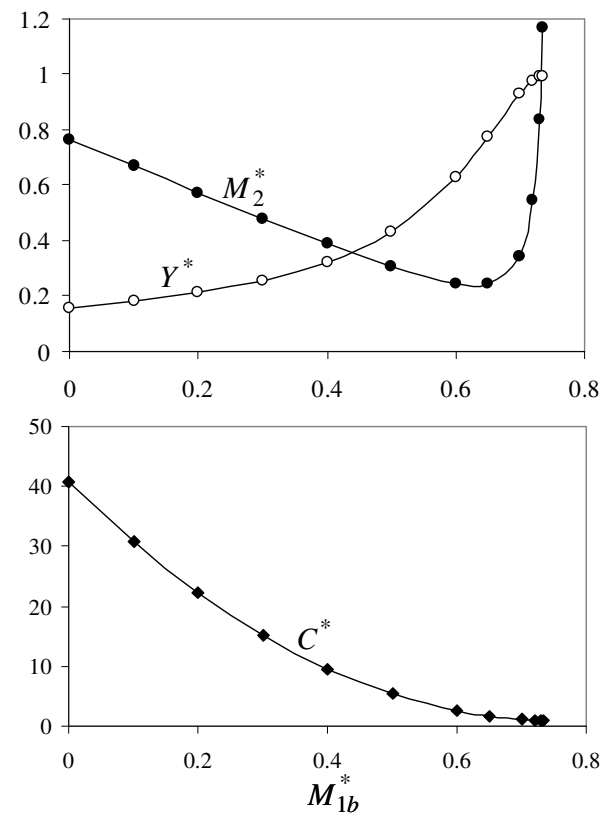

Fig. 5. Dimensionless plots of $M_{2}^{*}, Y^{*}$ and $C^{*}$ versus $M_{1 b}^{*}$, for $\alpha=1$ (tube of uniform inside cross section) and wave period $T^{*}=10$. Maximum absorbed power $\bar{P}^{*}=1$ is attained for all plotted points.

motion between bodies 1 and 2; (ii) $M_{2}^{*}$ (mass plus added mass of body 2); (iii) PTO damping coefficient $C^{*}$. For all plotted points, it is $\bar{P}^{*}=\bar{P} / \bar{P}_{\max }=1$, since maximum capture width $\lambda / 2 \pi$ ( $\lambda=$ wavelength) is attained by the maximization process. We note that the wave energy is absorbed solely from the motion of body $1 a$ (bodies $1 b$ and 2 are assumed far away from the free surface). So $X *$ is the same as for a single body $1 a$ optimally reacting against the bottom; it depends only on $T^{*}$ and is independent of the optimal pair $\left(M_{1 a}^{*}, M_{2}^{*}\right)$. It is well known (see e.g. [17]) that, for a single heaving body, maximum absorbed power is attained for oscillation amplitude
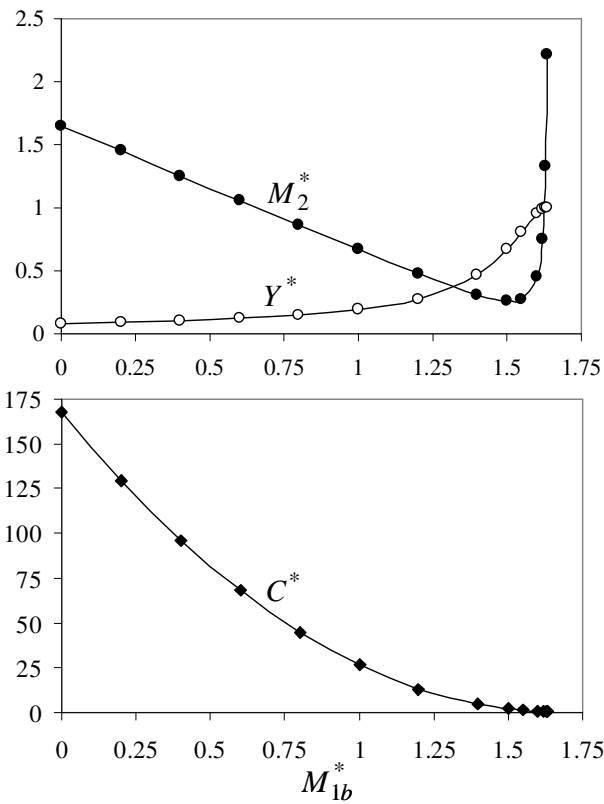

Fig. 6. As in Fig. 5, for $T^{*}=12$.
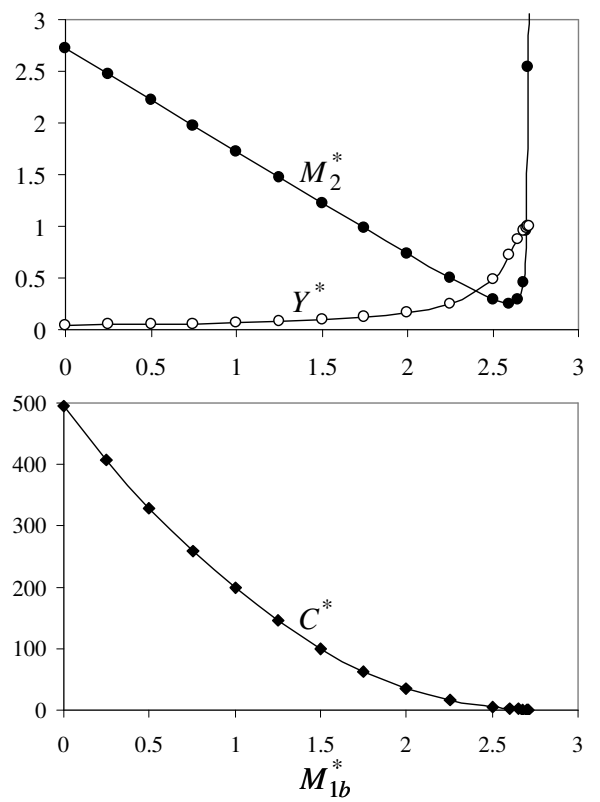

Fig. 7. As in Fig. 5, for $T^{*}=14$. 
$X_{\mathrm{opt}}=F_{e}(2 i \omega B)^{-1}$, which, for axisymmetric floating body $1 a$, can be written in dimensionless form as

$$
X_{\mathrm{opt}}^{*}=(2 \pi)^{-7 / 2} B^{*-1 / 2} T^{* 3},
$$

where $B^{*}=B\left(\rho \pi a^{3} \omega\right)^{-1}$. We recall that $B^{*}$ is a function of $T^{*}$. The optimal value $X_{\mathrm{opt}}^{*}$, for buoy $1 a$, is plotted versus $T^{*}$ in Fig. 8 and can be seen to increase rapidly with $T^{*}$ in the plotted range.

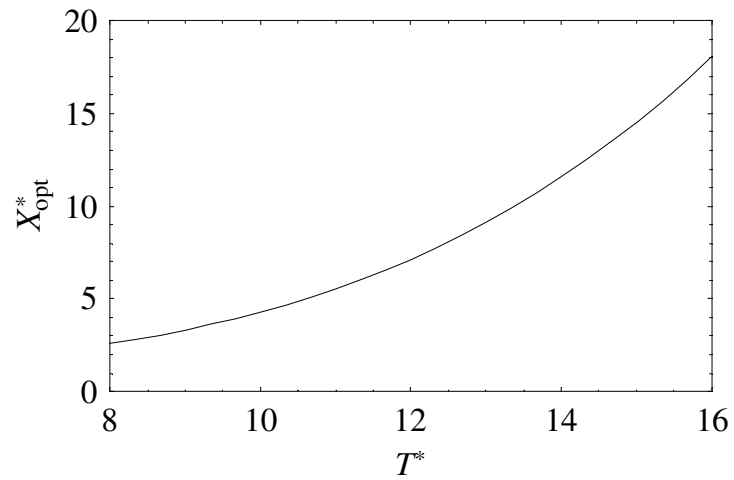

Fig. 8. Dimensionless plot of optimal oscillation amplitude $X_{\mathrm{opt}}^{*}$ of buoy 1 a versus wave period $T^{*}$.

In Figs. 5-7, the curve for $M_{2}^{*}$ exhibits a minimum, for a value of $M_{1 b}^{*}$ that increases with $T^{*}$. To the right of this point, $M_{2}^{*}$ increases to infinity. In the limiting situation when $M_{2}^{*}=\infty$, we have what is equivalent to a single body $(1 a+1 b)$ reacting against the bottom $\left(Y^{*}=1\right)$, for which case the optimal conditions are well known (see e.g. [17]): $M_{1 a}+M_{1 b}=\rho g S / \omega^{2}$ and $C=B$ (i.e. $C^{*}=1$ ). In this limiting situation, it is

$$
M_{1 b}^{* *}=\frac{\rho g S}{\omega^{2} m_{1 a}}-\frac{\mu_{1 a}}{m_{1 a}}-1,
$$

which, for the special case of the cylindrical buoy with conical bottom considered here, becomes $M_{1 b}^{* *}=0.02124 T^{2}$. $-0.8387 \mu_{1 a} / m_{1 a}-1$ (Note that, like $B_{1 a}^{*}, \mu_{1 a} / m_{1 a}$ is also function of $T^{*}$.) This equation is plotted in Fig. 9. It is $M_{1 b}^{* *}=0$ for $T^{*}=7.940$, which means that body $1 a$, if isolated, would be perfectly tuned to this wave period.

We note that $Y^{*}$ increases and $C^{*}$ decreases with increasing $M_{1 b}^{*}$, with (as should be expected) $Y^{*} \rightarrow 1$ and $C^{*} \rightarrow 1$ as the limiting case $M_{2}^{*}=\infty$ is approached. Since, for the plotted points, it is $\bar{P}^{*}=1$, it can easily be shown that, for fixed $T^{*}$ and varying $M_{1 b}^{*}, C^{*}$ is proportional to $Y^{*-2}$. The dimensionless amplitude of the PTO force oscillations $C * Y *$

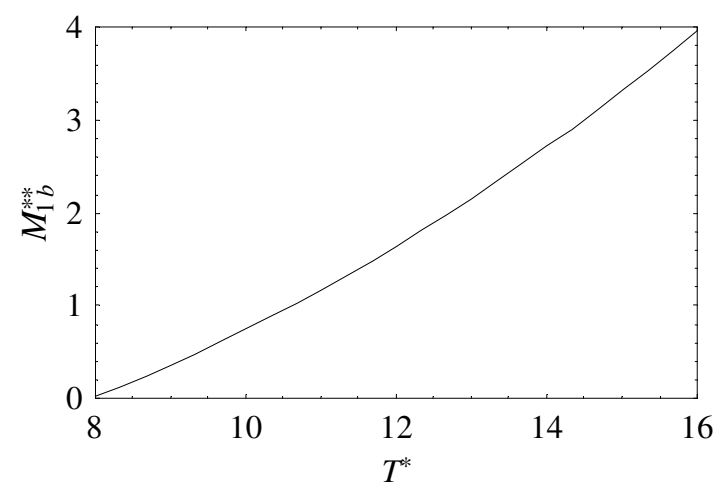

Fig. 9. Plot of $M_{1 b}^{* *}=0.02124 T^{2}-0.8387 \mu_{1 a} / m_{1 a}-1$ for the considered cylindrical buoy with conical bottom.

is proportional to $C^{* 1 / 2}$ (or to $Y^{*-1}$ ) and, as seen in Figs 5-7, increases rapidly with decreasing $M_{1 b}^{*}$.

3.2 Tube of non-uniform cross section $\alpha>1$. If $\alpha>1$, it is $A_{2}>A_{1}$, and the flow in the acceleration tube is no longer uniform. For this reason, the presence and inertia of water inside the tube can no longer be represented simply by a solid body and its mass. Besides, the force $f_{t}$ on the conical parts of the tube inner surface is non-zero and may be significant. So (unlike if $\alpha=1$ ), more than one parameter is now required to describe the water contained in the tube and the forces associated with it. In addition to $\alpha$, we use, as parameters, the tube diameter $D_{2}$, the length $b_{1}$ of the central part of the tube, the total tube length $L$, and the half-angle

$$
\beta=\arctan \left[\frac{D_{2}}{2 b_{2}}\left(1-\alpha^{-1}\right)\right]
$$

of the inner conical walls of the tube. As before, we introduce dimensionless quantities $D_{2}^{*}=D_{2} / a, b_{1}^{*}=b_{1} / a$ and $L^{*}=L / a$ (where $a$ is the floater radius). Since the length $b_{3}+b_{4}$ of the tube segments of diameter $D_{2}$ should be non-negative, the following restriction applies

$$
L^{*} \geq b_{1}^{*}+D_{2}^{*}\left(1-\alpha^{-1}\right) \cot \beta
$$

Figure 10 shows results for $T^{*}=10$. A geometry representative of an IPS buoy with piston was chosen: $b_{1}^{*}=0.533, \beta=\pi / 6$ and $\alpha=D_{2} / D_{1}=1.25$. Results for $\alpha=1$ are also shown for comparison. For all plotted points, the maximization procedure yielded $\bar{P}^{*}=1$. It can be seen that, for fixed $T^{*}$ and $M_{1 b}^{*}$, a larger value of $\alpha$ (1.25 as compared with 1) results in larger tube length $L^{*}$, larger piston displacement amplitude $Y^{*}$ and smaller PTO damping coefficient.

If a hydraulic turbine is to be used instead of a piston, a much larger value of $\alpha$ should be chosen as well as a much 
smaller value of $b_{1}^{*}$. Figure 11 shows results for $\alpha=4$, $b_{1}^{*}=0.2$ and $\beta=\pi / 4$. Two wave periods are represented: $T^{*}=10$ and 12. As before, the maximization procedure yielded $\bar{P}^{*}=1$ for all plotted points. The differences with respect to the case $\alpha=1$ (tube of uniform inner cross section) are now much more marked, especially on what concerns $Y^{*}$ and $C^{*}$.
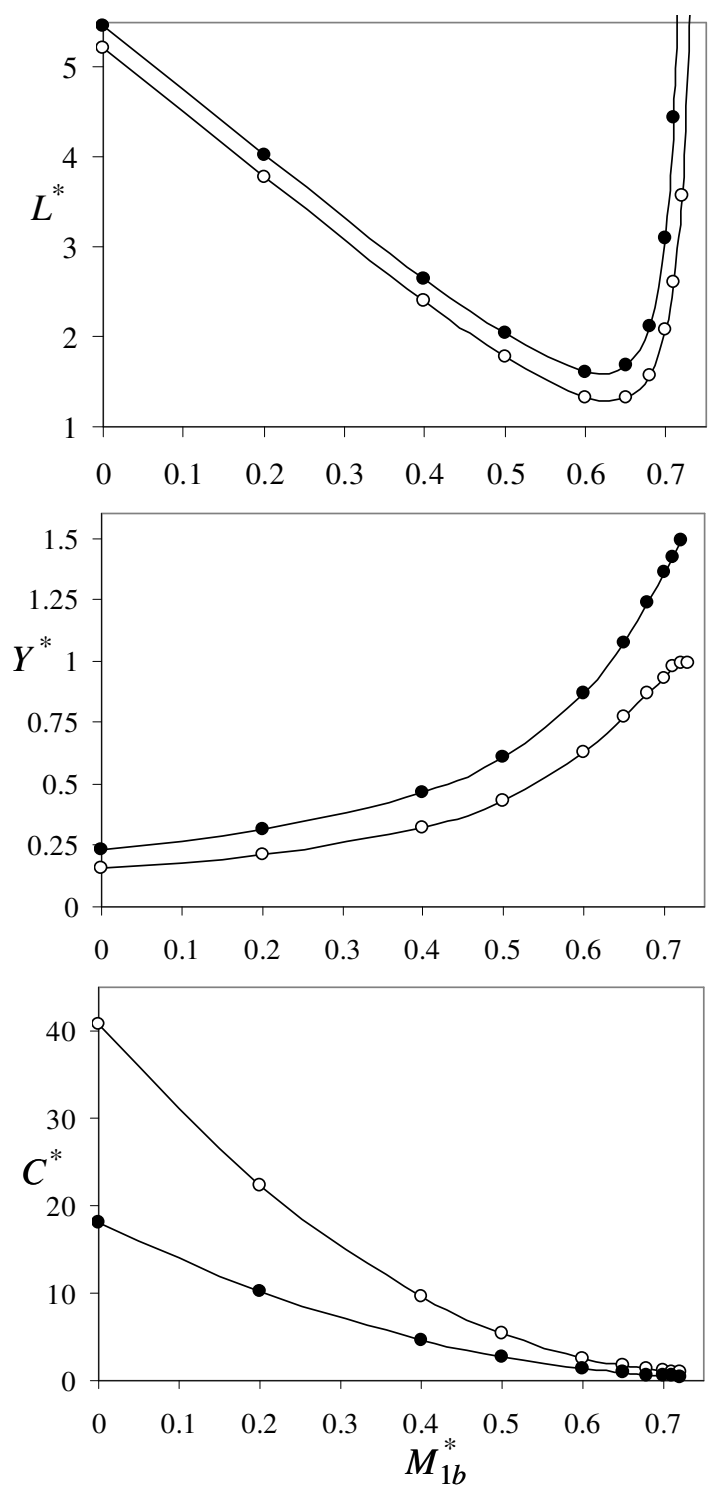

Fig. 10. Dimensionless plots of $L_{2}^{*}, Y^{*}$ and $C^{*}$ versus $M_{1 b}^{*}$, for $\alpha=1$ (open symbols) and $\alpha=1.25$ (closed symbols). Tube geometry: $b_{1}^{*}=0.533, \beta=\pi / 3$. Wave period $T^{*}=10$. Maximum absorbed power $\bar{P}^{*}=1$ is attained for all plotted points.
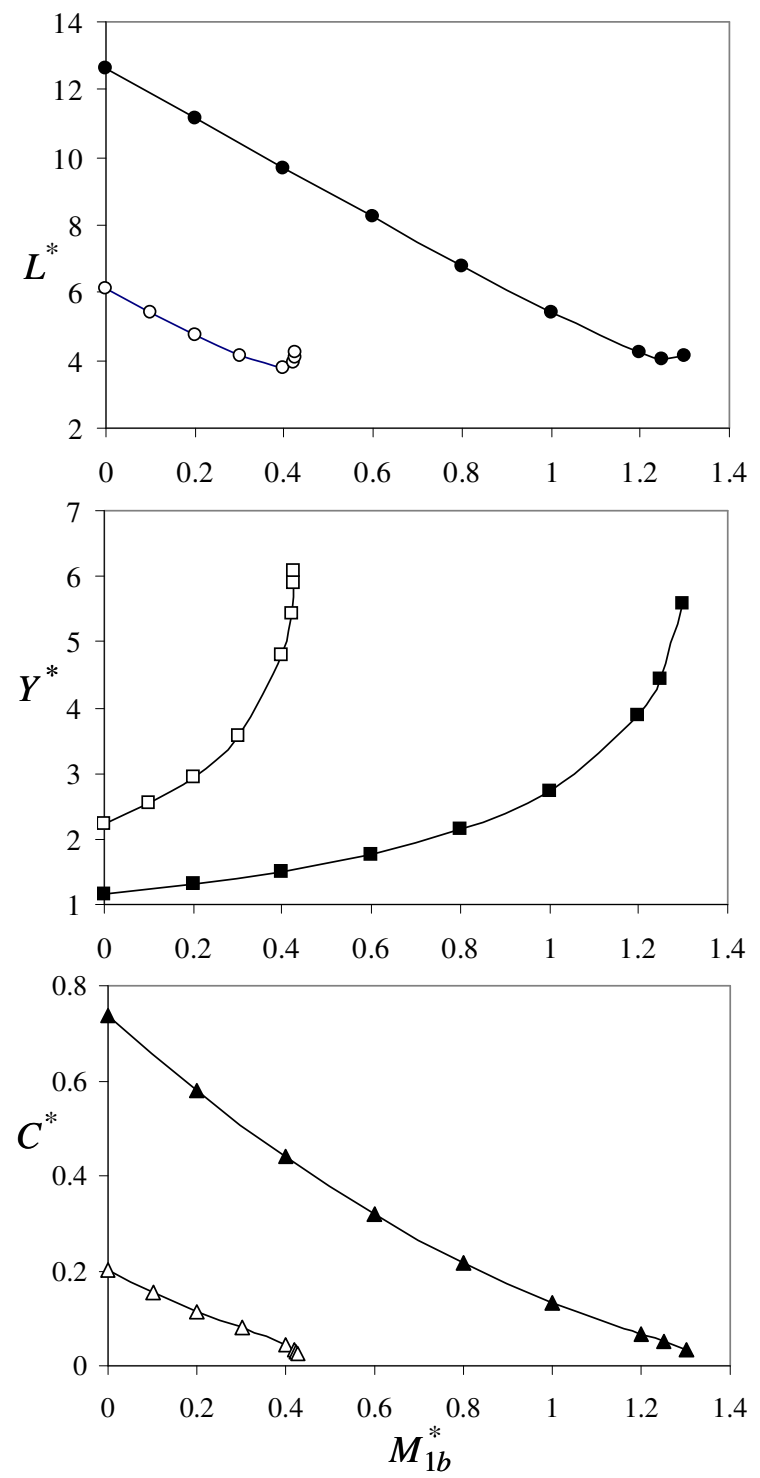

Fig. 11. Dimensionless plots of $L_{2}^{*}, Y^{*}$ and $C^{*}$ versus $M_{1 b}^{*}$. Tube geometry: $\alpha=4, b_{1}^{*}=0.2, \beta=\pi / 4$. Wave periods $T^{*}=10$ (open symbols) and 12 (closed symbols). Maximum absorbed power $\bar{P}^{*}=1$ is attained for all plotted points.

It is interesting to examine in detail the curves $L^{*}$ versus $M_{1 b}^{*}$ plotted in Fig. 11 for $\alpha=4$ and compare them with the curves of $M_{2}^{*}$ versus $M_{1 b}^{*}$ plotted in Figs 5 and 6 for $\alpha=1$. We recall that it is $\bar{P}^{*}=1$ for all plotted points in these figures. In all cases, the curves exhibit a minimum. But, while in Figs 6 and 7 the curves to the right of this point rise to $M_{2}^{*}=\infty$ (in the limit representing the single body $1 a+1 b$ reacting against the sea bottom), in Fig. 11 the curves are of finite extent: the condition $\bar{P}^{*}=1$ cannot be maintained beyond the last plotted 
point, well short of $L^{*}=\infty$. In what follows we attempt to explain this different behaviour. Let us denote by $V_{1}$ the relative flow velocity, and by $U_{1}=V_{1}+W$ the absolute flow velocity, in the central part of the tube where the crosssectional area is $A_{1}$ (we recall that $W$ is the velocity of the buoy-tube set). Then, in the part of the tube where the area is $A_{2}=\alpha^{2} A_{1}$, the relative flow velocity is $V_{2}=\alpha^{-2} V_{1}$ and the absolute flow velocity is $\alpha^{-2} V_{1}+W=U_{2}$ (say). If we fix the length $b_{1}$ of the central part of the tube and let $b_{3}+b_{4} \rightarrow \infty$ (i.e. $L \rightarrow \infty$ ), then, because of the inertia of the very large volume of enclosed water, it will be $U_{2} \rightarrow 0$ and, consequently, $V_{1} \rightarrow-\alpha^{2} W$ and $U_{1} \rightarrow-W\left(\alpha^{2}-1\right)$. We may say that, for $\alpha>1$, even if the tube length becomes very large, the absolute velocity of the piston (or of the flow admitted to the hydraulic turbine) does not vanish and its direction is opposite to the velocity direction of the buoy-tube pair. This, together with the fact that the force $f_{t}$ on the inner surface of the tube is non-zero, explains why, if $\alpha>1$, from the viewpoint of device performance, the water contained in a very long acceleration tube $\left(b_{3}+b_{4} \rightarrow \infty\right)$ is not equivalent to a solid body of infinite mass.

\section{Conclusions}

A frequency domain analysis of the IPS hydrodynamics, combined with a one-dimensional model of the (in general non-uniform) unsteady flow inside the acceleration tube, has been developed and used to assess the performance of the device in regular incident waves. In spite of somewhat drastic simplifying assumptions concerning the wave field interference between buoy and tube, the obtained results are believed to be significant.

If the cross section of the acceleration tube is non-uniform (which could be dictated by practical reasons, namely to limit the piston excursion or to allow a hydraulic turbine to be installed), the flow inside the tube is also non-uniform, and the inertia of the enclosed water cannot be represented by that of a solid body. Besides, apart from the axial force on the piston (or on the hydraulic turbine), the extra axial force on the noncylindrical inner walls of the tube has to be accounted for. In such situations, the dynamics of the IPS buoy can no longer be theoretically modelled as Falnes [18] did for a two-body heaving system or as done in [16] for the IPS buoy.

In regular waves, it was found that, for a given buoy and given tube diameter $D_{2}$ and diameter ratio $\alpha$, maximum wave energy absorption (equal to what can be achieved by an axisymmetric heaving body) is attained by an infinite number of combinations of mass $M_{1 b}$, tube length $L$ and PTO damping coefficient $C$. If $D_{2}$ and $M_{1 b}$ are kept fixed and $\alpha$ allowed to increase above unity (or $D_{1}$ to decrease below $D_{2}$, i.e. a more marked nozzle effect), the conditions for maximum energy absorption require a longer tube, a longer piston excursion and a smaller damping coefficient.

\section{Acknowledgement}

The work reported here was partly supported by the Portuguese Foundation for Science and Technology under contracts POCI-SFA-46, PTDC/EME-MFE/66999/2006 and PTDC/EME-MFE/103524/2008. Co-author JCCH thanks Programa Ciência 2007 for financial support.

\section{References}

[1] Falcão, A.F. de O., 2010. "Wave Energy Utilization: a Review of the Technologies," Renew. Sust. Energy Rev., 14, pp. 133-144.

[2] Masuda Y. 1971. "Wave-Activated Generator," Int. Colloq. Exposition Oceans, Bordeaux, France.

[3] Masuda, Y. 1986. "An Experience of Wave Power Generator through Tests and Improvement." In: Evans, D.V., Falcão, A.F. de O. (editors), Hydrodynamics of Ocean Wave-Energy Utilization, Springer-Verlag, Berlin, p. 445-452.

[4] Noren, S.A., 1981. "Plant for Utilizing Kinetic Energy." US Patent No. 4,277,690. (Original Swedish Patent No. 7808679, 1978.)

[5] Noren, S.A., 1988. "Apparatus for Recovering the Kinetic Energy of Sea Waves.” US Patent No. 4,773,221. (Original Swedish Patent No. 8104407, 1981.)

[6] Cleason, L., Forsberg, J., Rylander, A., Sjöström, B.O., 1982. "Contribution to the Theory and Experience of Energy Production and Transmission from the BuoyConcept." In: Proc. $2^{\text {nd }}$ Int. Symp. Wave Energy Utilization, Trondheim, Norway, p. 345-370.

[7] Fredriksson, G., Sjöström, B.O., Cleasson, L., Forsberg, J., 2000. "Wave Energy Converter." US Patent No. 6,140,712. (Original Swedish Patent No. 9601638, 1996).

[8] Weinstein, A., Fredrikson, G., Parks, M.J., Nielsen, K., 2004. "AquaBuOY, the Offshore Wave Energy Converter Numerical Modelling and Optimization." In: Proc. MTTS/IEEE Techno-Ocean '04 Conf., Kobe, Japan, vol. 4, p. 1854-1859.

[9] Salter, S.H., Lin, C.P., 1998. "Wide Tank Efficiency Measurements on a Model of the Sloped IPS Buoy". In: $3^{\text {rd }}$ European Wave Energy Conf., Patras, Greece, p. 200-206.

[10] Payne, G.S., Taylor, J.R.M., Bruce, T., Parkin, P., 2008. "Assessment of Boundary-Element Method for Modelling a Free-Floating Sloped Wave Energy Device. Part 1: Numerical Modelling," Ocean Eng. 35, pp. 333-341.

[11] Payne, G.S., Taylor, J.R.M., Bruce, T., Parkin, P., 2008. "Assessment of Boundary-Element Method for Modelling a Free-Floating Sloped Wave Energy Device. Part 2: Experimental Validation," Ocean Eng. 35, pp. 342-357.

[12] Gomes, R.P.F., Henriques, J.C.C., Gato, L.M.C., Falcão, A.F.O., 2010, "IPS 2-Body Wave Energy Converter: 
Acceleration Tube Optimization," Int. J. Offshore Polar Eng., 20, pp. 247-255.

[13] Noble, B., 1958. Methods Based on the Wiener-Hopf Technique for the Solution of Partial Differential Equations. Pergamon Press, London.

[14] Evans, D.V., 1978. "The Oscillating Water Column WaveEnergy Device,” J. Inst. Maths Applics, 22, pp. 423-433.

[15] Munson, B.R., Young, D.F., Okiishi, T.H., 1994. Fundamentals of Fluid Mechanics. Second ed., Wiley, New York.
[16] Falcão, A.F. de O., Justino, P.A.P., Henriques, J.C.C., André, J.M.C.S., 2008. "Modelling and Control of the IPS Buoy." In: Proc. $2^{\text {nd }}$ Int. Conf. Ocean Energy, Brest, France.

[17] Falnes, J., 2002. Ocean Waves and Oscillating Systems. Cambridge University Press, Cambridge.

[18] Falnes, J., 1999. "Wave-Energy Conversion through Relative Motion between Two Single-Mode Oscillating Bodies," J. Offshore Mech. Arct. Eng., 121, pp. 32-38. 